\title{
Nontuberculous Mycobacterial Lung Disease: An Emerging Disease in the Elderly
}

\author{
Erin Epson ${ }^{1, *}$ and Kevin Winthrop ${ }^{1,2,3}$ \\ ${ }^{1}$ Division of Infectious Diseases, Oregon Health \& Science University, Portland, OR, USA \\ ${ }^{2}$ Department of Ophthalmology, ${ }^{3}$ Division of Public Health and Preventive Medicine (joint appointment), Oregon \\ Health \& Science University, Portland, OR, USA
}

\begin{abstract}
Multiple recent population-based studies document the rising prevalence of lung disease caused by the environmentally ubiquitous nontuberculous mycobacteria (NTM), particularly among older women. The reasons for this changing epidemiology are unclear, and the specific predisposing conditions and pathophysiologic mechanisms underlying the development of NTM lung disease among affected individuals are poorly understood. Progress in our understanding of this disease has been hampered by the lack of animal models for NTM lung disease. Various systemic immune defects, structural lung diseases, and a constellation of factors among a subset of post-menopausal women with a distinct body morphotype have all been evaluated among affected individuals, yet the majority of NTM lung disease remains incompletely explained. This review summarizes the available literature describing NTM as an emerging pathogen particularly among the aging population, including the changing epidemiology, clinical features of NTM lung disease, and proposed pathophysiologic mechanisms underlying disease susceptibility and manifestations particularly among the elderly. The rising prevalence of NTM lung disease and significant gaps in our understanding and tools for study highlight this disease as an important subject of further research.
\end{abstract}

Keywords: Nontuberculous mycobacteria, lung disease, aging.

\section{SOMETHING IN THE WATER}

The nontuberculous mycobacteria are a large and diverse group of microorganisms exhibiting varied pathogenicity in humans. There has been a dramatic expansion in recognized NTM species over the past few decades largely as a result of advances in molecular techniques capable of differentiating distinct mycobacterial species, with more than 140 species of NTM now identified [1]. Of these, Mycobacterium avium complex (MAC), M. kansasii, and M. abscessus (one of the group termed "rapidly growing mycobacteria") rank among the most commonly implicated NTM in causing NTM lung disease in the United States and most regions of the world [1]. The particular NTM species causing lung disease vary in some regions, however. In parts of Canada, the United Kingdom and central Europe, M. xenopi is the third most common cause of NTM lung disease, whereas in northern Europe, $M$. malmoense ranks behind MAC as the second most common cause of NTM lung disease [2,3]. Additional mycobacterial species are likely to be identified in the coming years, and scientists and clinicians will be faced with the tasks of further characterizing them and determining their clinical significance. For example, $M$. abscessus is beginning to be subspeciated into 3 distinct species (M. abscessus sensu stricto, M. bolletti, and M. massiliense), which has clinical and therapeutic implications in that these subspecies appear to have distinct antimicrobial susceptibility features [4].

*Address correspondence to this author at the Division of Infectious Diseases Oregon Health \& Science University, 3181 SW Sam Jackson Park Road L457, Portland OR 97239-3098, USA; Tel: 503 494-7735;

Fax: 503 494-4264; E-mail: mcnelley@ohsu.edu
The NTM are ubiquitous environmental organisms found in soil and both natural and treated water in diverse geographic areas throughout the world [5, 6]. NTM acquisition by humans is believed to occur through environmental exposure, and there is increasing suspicion that biofilms in municipal water sources may represent a significant mode of human NTM exposure via aerosolization. A recent study utilizing ribosomal RNA sequencing techniques demonstrated that sequences representative of NTM were highly enriched in biofilms from 45 showerhead sites around the United States, $>100$-fold above background water contents [7]. Households with water heater temperatures $</=125$ degrees $\mathrm{F}$, and those with water supplied from public or private water systems serving multiple households, have been found to be more likely to harbor NTM [8]. A few reports have demonstrated the same genotype of MAC isolate in both plumbing sources and those obtained from humans with MAC lung disease $[9,10]$. Because of this ubiquity and widespread means human exposure, the NTM are a potential public and environmental health threat for those at risk of developing disease.

\section{CHANGING EPIDEMIOLOGY}

NTM were first recognized and described as human pathogens during the 1950s among a small subset of patients being treated at tuberculosis (TB) sanitaria, who were not improving on traditional anti-tuberculosis therapies [11, 12]. These individuals had disease caused by acid-fast organisms other than Mycobacterium tuberculosis. They tended to be 
older, and more likely to be Caucasian and have underlying lung disease, compared to those with TB.

Over subsequent decades, coinciding with a substantial decline in TB rates in North America, multiple institutional case series and anectodal reports suggested that the incidence of lung disease due to NTM was rising [1]. Yet determining the actual incidence and prevalence of NTM disease has been problematic in part because unlike TB, NTM disease is not reportable to public health authorities. Additionally, because NTM are ubiquitous in the environment and thus common colonizers or contaminants of respiratory specimens, the determination of lung disease rates from NTM isolation frequency data requires clinical context.

The first United States national survey based upon NTM isolates submitted to state laboratories from 1981-1983 estimated NTM disease prevalence at 1.8 per 100,000 persons [13]; males predominated and the mean age was 57 years among those with NTM lung disease. Population-based studies confirming suspicions of rising prevalence and associated risk factors for NTM lung disease had been lacking, until the recent publication of several reports documenting such increases as well as important changes in the epidemiologic features of this disease. In Ontario, Canada, NTM isolation prevalence increased from 9.1 per 100,000 population in 1997 to 14.1 per 100,000 population in 2003 [14]. In Oregon, pulmonary NTM disease prevalence in 2005-2006 was found to be 5.6 per 100,000 persons, with significantly higher rates among women (6.4 cases per 100,000 population) versus men (4.7 cases per 100,000 population) [15]. Prevalence was highest in this study among those aged $>50$ years, at 15.5 cases per 100,000 population. An analysis of clinical and radiographic data among a population-based subset of this group, from which NTM lung disease estimates were based upon the application of American Thoracic Society (ATS)/Infectious Diseases Society of America (IDSA) diagnostic criteria, confirmed these findings: the 2-year period prevalence was 8.6/100,000 persons, and 20.4/100,000 in those at least 50 years of age, with the majority (59\%) occurring among women [16]. Similarly, a study evaluating NTM lung disease at 4 geographically diverse healthcare delivery systems in the US demonstrated that between 2004-2006, average annual site-specific prevalence ranged between 1.46.6 per 100,000 , with significant increases at the 2 sites where trends were studied [17]. There was a 1.1 to 1.6 higher prevalence among women relative to men in this study, and substantial increases in annual prevalence among persons aged greater than or equal to 60 years, from 19.6 per 100,000 to 26.7 per 100,000 population over this time-period (Fig 1). Thus, NTM lung disease can legitimately be considered an emerging infection in the aging population, particularly among women.

\section{CLINICAL FEATURES}

Clinical manifestations of NTM disease in humans range from disseminated infection in immunocompromised individuals, cervical and thoracic lymphadenitis in children, skin, soft-tissue and bone infections, and chronic lung disease [1]. Chronic lung disease represents the most common clinical manifestation of NTM infection $[13,18]$.

Various structural lung diseases have been described in

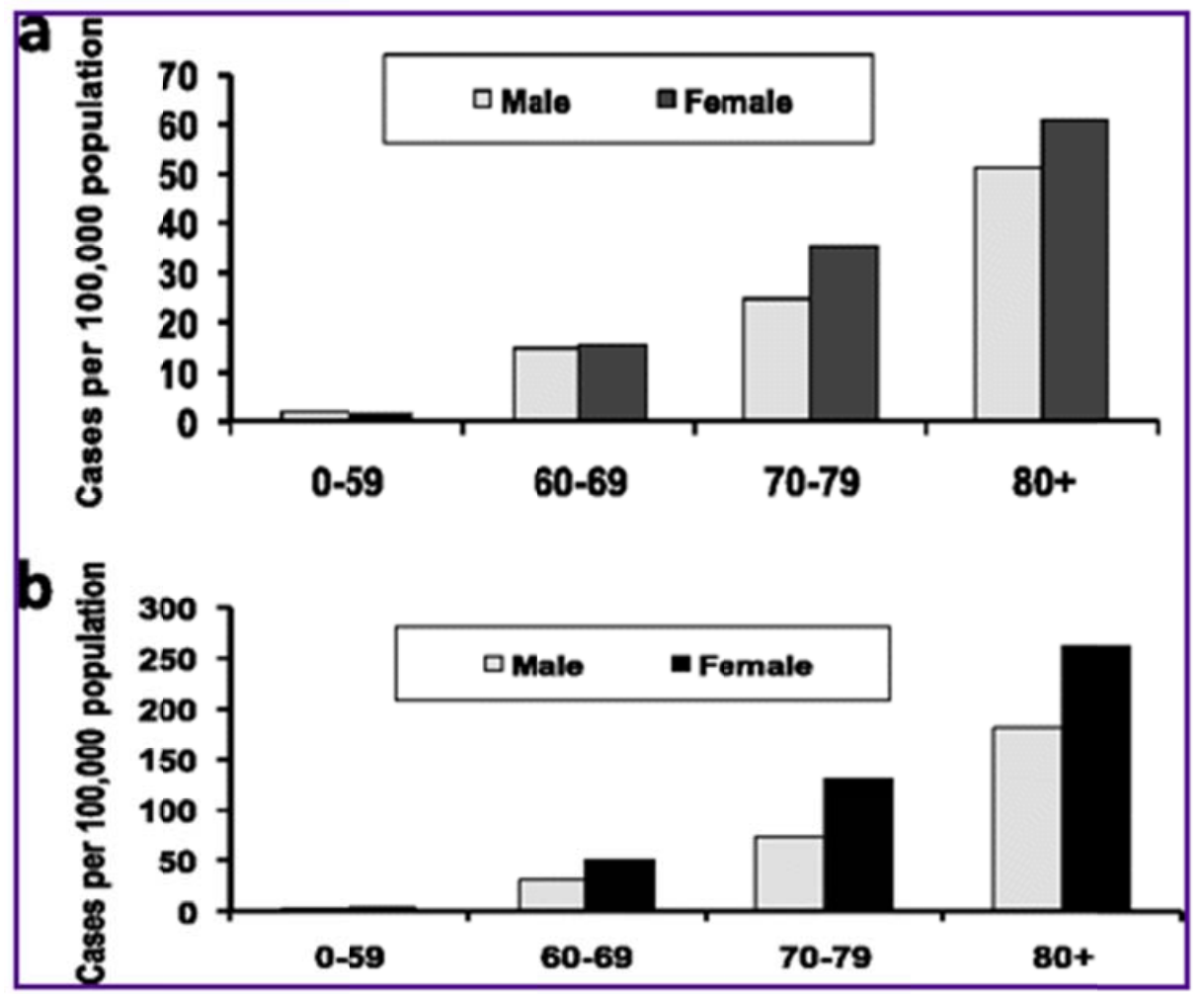

Fig. (1). NTM lung disease is a disease of older individuals, predominantly women. (A) Average annual prevalence by age and sex. Group Health and Kaiser Permanente Southern California, combined, 2004-2006. (B) Cumulative incidence by age and sex. Group Health and Kaiser Permanente Southern California, combined, 2004-2006. (Prevots, AJRCCM 2010). 
association with NTM lung disease. These include chronic obstructive pulmonary disease (COPD), bronchiectasis, cystic fibrosis (CF), prior TB, silicosis, pneumoconiosis, and pulmonary alveolar proteinosis [13, 19-24]. NTM lung disease also occurs in older, post-menopausal, non-smoking women without previous lung disease [25]. A distinct body morphotype among such women has been described [26, 27], characterized by thin body habitus, mitral valve prolapse and thoracic cage abnormalities including scoliosis and pectus excavatum.

Symptoms and signs of NTM lung disease are nonspecific and variable, and frequently co-exist with symptoms caused by underlying lung diseases [1]. Virtually all patients experience chronic or recurring cough; other symptoms variably include sputum production, dyspnea, hemoptysis, chest pains, fevers, malaise and weight loss. Chest radiographic features can be characterized by either: upper lobe fibrocavitary changes (similar to TB) that are most common among men with underlying lung disease; or centrilobular nodules with tree-in-bud pattern and bronchiectasis, which are the typical features among postmenopausal women with this disease (Fig. 2). These radiographic types are not exclusively confined to one sex or the other, however, and both fibrocavitary and nodular bronchiectasis can occur in one patient [28]. Histopathologically the nodular radiographic findings correspond to bronchiolar and peribronchiolar inflammation, and granuloma formation [29].

\section{PATHOGENESIS AND SUSCEPTIBILITY}

\section{Host Immune Response to Mycobacterial Infection}

The study of host immune responses in NTM lung disease has been hampered by the lack of good animal model systems for this disease. Mice can be infected with NTM via the pulmonary route but do not develop endobronchial dis-

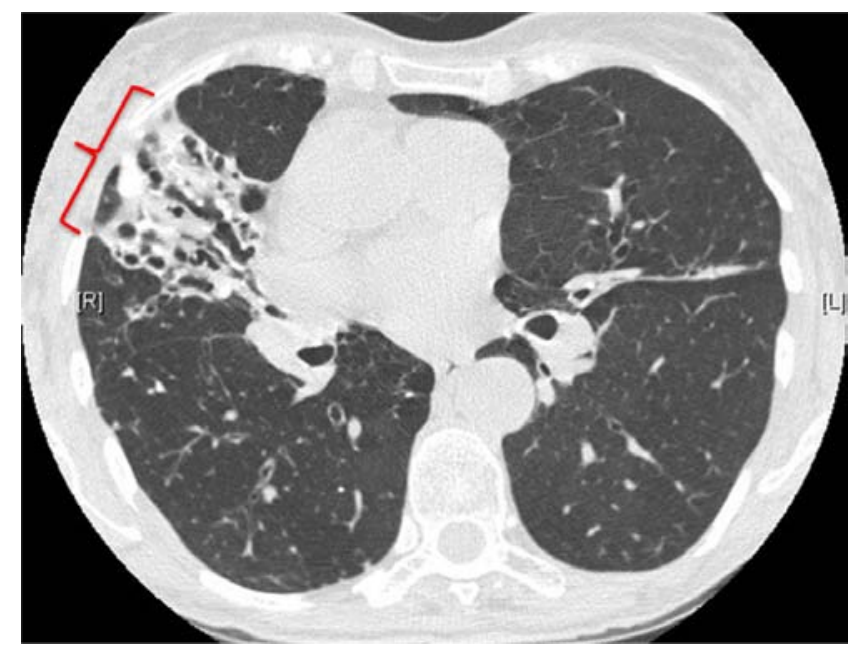

Fig. (2). Computed tomography of the chest of a 74 year-old woman with recurrent MAC lung disease. This representative image depicts abnormally dilated and inflamed airways characterizing bronchiectasis involving the right middle lobe with surrounding opacification due to infiltration of inflammatory cells and fluid (indicated by bracket). The patient was being treated with antimycobacterial drug therapy, and was scheduled for right middle lobectomy surgery. ease as seen in humans [30]. As such, our understanding of the host immune pathways involved in NTM infection has largely been derived from the study of humans and genedeficient animals that develop disseminated mycobacterial disease [31].

Like M. tuberculosis, the NTM are ingested by macrophages. Binding of mycobacterial lipoproteins to macrophage Toll-like receptors (TLRs) is thought to be important in initiating both innate and adaptive immune responses to pathogenic mycobacteria [32]. TLR stimulation induces production of interleukin (IL)-12 which in turn binds its receptor on $\mathrm{T}$ lymphocytes and natural killer cells, and via the signal transducer and activation of transcription 4 (STAT4) signaling pathway up-regulates production of interferon (IFN)- $\gamma$. IFN- $\gamma$ binds its receptor on the surface of many inflammatory cells, and via the Janus kinase (JAK)-STAT pathway induces expression of various genes mediating both innate and adaptive immune functions important in the response to mycobacteria, including: enhancement of intracellular mycobacterial killing through induction of free radical formation via inducible nitrogen oxide synthase (iNOS); upregulation of major histocompatability complex (MHC) class I and II for antigen presentation to CD8+ cytotoxic T lymphocytes and CD4+ helper $\mathrm{T}$ lymphocytes, respectively; promotion of differentiation of T-helper lymphocytes into the pro-inflammatory Th1 phenotype; and promotion of other inflammatory cytokine synthesis including tumor necrosis factor (TNF)- $\alpha$ and IL-12. Thus, a positive feedback loop exists between IFN- $\gamma$ and IL-12 production, connecting both innate and adaptive immune responses and mediating critical elements for control of mycobacterial infection.

\section{Systemic Immune Defects and NTM Disease}

Given the environmental ubiquity of NTM, either intense exposure or defects in systemic and/or local host immunity are thought likely to be required for human disease to occur [33]. Indeed, disseminated NTM infection is observed in HIV-infected patients after the CD4+ T-lymphocyte count decreases below 50 cells $/ \mu \mathrm{L}$. Additionally multiple case reports and series of HIV-negative individuals have described genetic syndromes of disseminated NTM infection, although not typically isolated lung disease, in association with mutations in IL-12, IFN- $\gamma$ and their receptors [34-38], as well as STAT1 deficiency [39]. Similarly there have been increasing reports of disseminated NTM infection among patients, especially of Asian ancestry, with acquired autoantibodies exhibiting a very high inhibitory activity against IFN- $\gamma[40$, 41]. Yet these particular rare mutations and immunologic abnormalities do not appear to explain the majority of disseminated NTM infections in HIV-negative individuals [33], nor do they appear to predispose affected individuals to isolated lung disease.

Although disseminated MAC infection occurs in patients with AIDS, isolated MAC lung disease is uncommon among these patients [42]. Impaired cell-mediated immunity also appears to impart an increased incidence of NTM infection among recipients of solid organ transplants (SOT) and hematopoietic stem cell transplants (HSCT). With the exception of lung transplant recipients, however, the most common sites of NTM infection among these patients are 
extrapulmonary [43]. There is a well established increased risk of TB among patients treated with TNF- $\alpha$ antagonists for inflammatory conditions such as rheumatoid arthritis and inflammatory bowel disease [44], and NTM infection has also been reported among patients receiving such therapies [45]; although lung disease is frequently reported among such patients, like with $\mathrm{TB}$, there is also a high rate of extrapulmonary infection in this setting.

Accordingly, many investigators have sought to identify specific mutations or immunologic defects among patients with isolated NTM lung disease. TLR-2 knockout mice have been reported to have increased susceptibility to M. avium infection [46], and the expression of TLR-2 among peripheral blood monocytes of 17 human patients with NTM lung disease was found to be impaired in comparison with healthy controls [47]. Genetic polymorphisms of the naturalresistance-associated macrophage protein 1 (NRAMP1), an ion transporter in the lysosomal membrane important during phagocytosis, were observed at higher frequencies in a study of 41 patients with NTM lung disease versus controls [48]. Significantly lower peripheral blood mononuclear cell (PBMC) production of cytokines IFN- $\gamma$, IL-12, and TNF- $\alpha$, have been described among among 29 patients with NTM lung disease versus healthy controls [49], as well as among 32 patients with NTM lung disease compared with patients with TB disease [50]. PBMC from patients with MAC lung disease have been found to produce higher levels of IL-10 but lower levels of IFN- $\gamma$ and TNF- $\alpha$ when compared with PBMC from healthy control subjects with positive delayed hypersensitivity skin test response to $M$. avium sensitin [51]. Similarly, lymphocytes isolated from a series of 5 patients with persistent or recurrent NTM lung disease were found to have significantly decreased IFN- $\gamma$ secretion in response to mitogen stimulation [52]. Detection of intracellular IFN- $\gamma$ in the presence of markedly suppressed extracellular release was demonstrated in 2 such individuals with recurrent NTM lung disease, suggesting either a defect in the IFN- $\gamma$ cellular transport pathway or the presence of an extracellular cytokine inhibitor as potential mechanisms for such defects in IFN- $\gamma$ secretion [53].

Yet other investigations have found no association between specific immunologic defects and NTM lung disease. In contrast to the previously described studies, a large cohort of 63 prospectively enrolled patients with NTM lung disease was found to have normal CD4(+), CD8(+), B, and natural killer cell numbers, and similar stimulated cytokine production compared with healthy control subjects (including the IFN- $\gamma /$ IL-12 pathway)[27], albeit these investigations utilized less specific antigenic stimuli versus some of the prior studies that did demonstrate differences [51]. Another evaluation of 8 women with MAC lung disease found no evidence of mutations affecting the IFN- $\gamma$ receptor or NRAMP1 [54]. Furthermore, intact granuloma formation capability has been demonstrated on histopathologic analysis of transbronchial lung biopsies among more than half of individuals with MAC lung disease in another series [55].

Vitamin D is increasingly recognized as having pleiotropic effects outside of calcium homeostasis and bone metabolism, such as an important role in immune system regulation, including the response to mycobacteria. There are numerous reports of associations between TB and vitamin D deficiency [56], as well as polymorphisms of the vitamin D receptor (VDR) [57], however the connection between VDR polymorphisms and NTM disease is less clear. Altered prevalence of three VDR polymorphisms was found in one series of patients with $M$. malmoense lung disease [58], however another study evaluated the same polymorphisms among patients with MAC lung disease and found no difference in prevalence compared with healthy controls [59].

\section{Chronic Lung Diseases, Local Inflammation, Defects in Airway Defenses, and Other Mediators of Susceptibility to NTM Lung Disease}

Indeed, the reasons for susceptibility to isolated lung disease caused by NTM remain poorly understood. Impaired local immune function due to underlying chronic lung disease with associated inflammation and mucosal damage likely both contribute to an increased propensity for NTM lung disease in some patients. In vitro studies have shown that NTM only adhere to damaged mucosal surfaces, and that infection with MAC is initiated when mycobacterial fibronectin attachment protein adheres to fibronectin within damaged mucosal surfaces [60, 61]; by comparison, MTB can adhere to intact mucosa. The propensity for some NTM to form biofilm, a characteristic also distinct from $M$. tuberculosis, may additionally contribute to the pathogenesis of NTM infection. A recent study demonstrated a significant relationship between the capacity for biofilm development and infection of various sites, including the respiratory tract, among clinically significant rapidly growing NTM isolates [62].

Bronchiectasis is a disease of the airways characterized by luminal dilatation resulting from chronic mucosal and submucosal injury, inflammation and remodeling. It is a common feature of NTM lung disease, and may be considered not only a sequela of NTM lung disease, but also a predisposing factor for NTM infection and disease progression. Airway damage due to persistent inflammation, as well as impaired clearance and abnormal composition of airway secretions, are all factors that compromise local immunity and provide a setting for persistent infection in bronchiectasis [63]. The pathogenesis of bronchiectasis is incompletely understood. Shared clinical features between idiopathic bronchiectasis and cystic fibrosis, as well as a frequent association between these conditions and NTM lung disease, have prompted evaluation into whether mutations in the cystic fibrosis transmembrane regulator (CFTR) gene play a role in the development of bronchiectasis [64-67] as well as NTM lung disease. Mutations in the CFTR gene and sweat chloride concentrations were prospectively evaluated in a series of 50 patients with idiopathic bronchiectasis and/or NTM lung disease, with half of the patients found to be either heterozygous or homozygous for CFTR mutations; 20\% actually met disease criteria for CF [68]. In another prospective study of 63 patients with NTM lung disease, 36\% were found to have at least one CFTR mutation [27]. A more recent analysis of 6 familial clusters of NTM lung disease found CFTR mutations among 5 of 12 patients in whom CFTR gene analysis was performed [69]. Yet to date, there has been no clear mechanistic connection established between single CFTR mutations and bronchiectasis or NTM 
lung disease, and these findings do not adequately explain the majority of NTM lung disease.

NTM lung disease is also seen among individuals with primary ciliary dyskinesia (PCD) [70], another pulmonary disease of impaired mucociliary clearance. Ciliary dysfunction was recently evaluated in a series of 32 patients with NTM lung disease none of which had confirmed PCD or CF prior to their presentation with NTM disease [71]. Nasal nitric oxide production was measured as a screen for ciliary or CFTR dysfunction. The mean nNO measurement for NTM lung disease patients was significantly lower than that of healthy controls, and nNO levels were consistent with primary ciliary dysfunction (PCD) in 9 (28\%) including 4 with confirmed PCD via compatible history and dynein abnormalities, and 5 with CFTR mutations.

Anomalous or deficient alpha1-antitrypsin (AAT) is another host factor that may confer increased susceptibility to NTM lung disease. AAT is a potent endogenous serine protease inhibitor, and individuals with homozygous $A A T$ gene abnormalities that result in severe AAT deficiency develop severe pulmonary emphysema due to unopposed host serine protease activity. Individuals with heterozygous AAT mutations may also have compromised AAT activity, and have been reported to have less severe pulmonary disease manifestations. In vitro experiments have suggested that AAT may additionally possess antimicrobial and antiinflammatory activity, and AAT replacement therapy has been reported to decrease the risk of respiratory infection among AAT deficient patients [72]. Accordingly, AAT protein abnormalities have been investigated as a risk factor for NTM lung disease caused by the rapidly growing mycobacteria (RGM). A recent study of a cohort of 100 patients with RGM lung disease referred for treatment at the National Jewish Medical and Research Center identified anomalous AAT proteins in $27 \%$ of the cohort, which is 1.6 times the estimated prevalence of such anomalous proteins in the general population $(\mathrm{p}=0.008)$ [73]. In companion in vitro experiments, both AAT and a synthetic inhibitor of serine proteases suppressed M. abscessus infection of monocyte-derived macrophages by up to $65 \%(\mathrm{p}<0.01)$. These findings will require further epidemiologic investigation to confirm the association between AAT abnormalities and NTM lung disease. Similar to CFTR mutations, AAT abnormalities do not appear to explain the majority of NTM lung disease.

\section{NTM Lung Disease Among Women with a Distinct Body Morphotype: the Lady Windermere Syndrome}

During the late 1980s, a series of patients with NTM lung disease without apparent predisposing factors was initially described; $76 \%$ of these patients had nodular radiographic features, and $81 \%$ were female [74]. Since that time additional features of this distinct clinical presentation have been described, including a predominance of radiographic findings isolated to lingula or right middle lobe [25]. It was hypothesized that the practice of voluntary cough suppression, such as among fastidious Victorian-era women such as Lady Windermere (prompting the name "Lady Windermere Syndrome" for this clinical entity) leads to trapping of secretions and local inflammation leading to NTM infection localized to these poorly draining regions of the lung. Yet this anatom- ic predilection as well as cough suppression have been inconsistently observed, and some have pointed out that patients with other conditions leading to impaired cough such as stroke or myopathy do not appear to have increased risk of NTM lung disease [75].

Thin body habitus as well as thoracic cage abnormalities of scoliosis and pectus excavatum are also observed among these women with nodular NTM lung disease and no other clearly apparent predisposing condition [26]. In a recent report of a series of 63 such patients with NTM lung disease evaluated at the National Institues of Health, 95\% were women, with mean age 59.0 +/- 9.8 years, $91 \%$ were Caucausian, and $68 \%$ were lifetime non-smokers [27]. Compared with age-matched controls, these patients' body mass index (BMI) was significantly lower and height significantly higher, and they also had higher rates of scoliosis (51\%), pectus excavatum (11\%), mitral valve prolapse $(9 \%)$ and CFTR mutations (36\%). Yet there were no chloride ion transport abnormalities associated with single CFTR mutations in these patients, and measurements of cytokine levels and lymphocyte subpopulations revealed no evidence of recognized innate or adaptive immune defects. Thus the pathophysiologic consequences of this constellation of body morphologic features remain poorly understood.

A very recent review summarizes clinical associations and experimental data to hypothesize 3 potential interrelated pathways to explain why older, post-menopausal women with thin body habitus and thoracic cage abnormalities would be more susceptible to NTM lung disease [76]: abnormal adipokine levels, relative estrogen deficiency, and abnormal expression of TGF- $\beta$ related to fibrillin anomalies similar to those seen in Marfan syndrome (MFS).

Chan and Iseman review experimental evidence supporting the immune function of fat. In particular, white adipose tissue in close proximity to lymph nodes demonstrates a bidirectional paracrine interaction between adipocytes and lymphocytes mediated by the hormones leptin and adiponectin [77]. Although the canonical function of leptin produced by white adipose tissue is to induce satiety, it can also behave as a cytokine with the ability to bias uncommitted $\mathrm{T}_{0}$ lymphocytes to the Th1 phenotype, which produces IFN- $\gamma$ [78]. There are few data on leptin and TB in humans, and no published studies of leptin levels in patients with NTM infections, however mice that are leptin deficient due to homozygous mutation of the gene encoding leptin, $o b$, have been found to be immunodeficient with regard to mycobacterial infection. When leptin-deficient $o b / o b$ mice were experimentally infected with $M$ tuberculosis, significantly greater numbers of tubercle bacilli were recovered from their lungs compared with wild-type mice, and there was a trend for lower survival among these mice at 30 weeks after infection [79]. Similarly, greater numbers of viable organisms were observed in the lungs of leptin-deficient versus wildtype mice infected with $\mathrm{M}$ abscessus [80], and the leptindeficient mice were noted to have lower influx of IFN- $\gamma$ producing $\mathrm{CD}^{+}$and $\mathrm{CD}^{+}$cells into their lungs. In contrast to leptin, adiponectin is secreted in inverse proportion to body fat, thus thinner individuals produce greater amounts of adiponectin. Adiponectin decreases TNF- $\alpha$, and increases IL10 production and IL-1 receptor antagonism, all of which 
have been shown to predispose animals to TB or MAC infection [81, 82]. Indeed adiponectin levels have been found to be significantly elevated in a consecutive series of 40 patients with MAC lung disease when compared with body mass index matched healthy controls [83]. Thus, decreased leptin and increased adiponectin in thin individuals are hypothesized as contributory factors for increased susceptibility to NTM.

The interaction between sex steroid hormones and noninfectious immune disorders as well as immune responses to infection has been described [84-87], and the proinflammatory properties of estrogen speculated to underlie the sex bias toward a substantially lower reporting of TB observed among women versus men [88]. Detailed evaluations of the role of sex hormones in the immune responses of individuals with mycobacterial disease are lacking, but in vitro experiments demonstrate that estrogen has multiple effects upon aspects of the immune response that are critical in the control of mycobacterial infection. For example, the binding of estrogen to estrogen receptors has been shown to augment phagocytic function and Fc receptor expression on macrophages [89], and estradiol has been shown to promote IFN- $\gamma$ production by natural killer cells [90]. Furthermore, female mice have been shown to be more resistant than males to infection with $\mathrm{M}$ intracellulare and $\mathrm{M}$ marinum [91, 92].

Estrogen deprivation, in turn, has been linked to several immunologic changes among post-menopausal women, including a decrease in B-lymphocytes and CD4 $+\mathrm{T}$ lymphocytes as well as a decrease in the cytotoxic activity of natural killer cells [93]. Estrogen deprivation among older, post-menopausal women has therefore been speculated to contribute to higher incidence of both chronic and infectious diseases among these individuals, and was also reviewed by Chan and Iseman as a potential factor in NTM disease susceptibility. In particular, decreased estrogen levels could be an intermediate step connecting lower leptin levels in thin individuals, which are associated with impaired release of gonadotropic hormones and secondary ovarian failure, with NTM disease susceptibility. Yet the experimental evidence linking estrogen signaling to NTM disease is less consistent and requires further clarification. In one report, ovariectomized mice were more susceptible to intratracheally administered MAC versus sham-treated mice, and reconstitution with exogenous estrogen restored the burden of bacilli in the ovariectomized mice to the same level as that in the sham-treated mice [94]. In vitro evaluation of peritoneal macrophages from estrogen receptor - $\alpha$ (ER- $\alpha)$ deficient animals, however, demonstrated that these macrophages produced more TNF- $\alpha$ in response to $M$ avium infection and had reduced bacterial load when compared with wild-type [95]. When ER- $\alpha$ deficient and wild type animals were fed diets rich in phytoestrogens, the ER- $\alpha$ deficient animals were found to have increased IFN- $\gamma$ production versus wild type animals [96].

In those NTM patients with morphologic features resembling the Marfan syndrome (ie, slender habitus with scoliosis, pectus excavatum and mitral valve prolapse), Chan and Iseman postulate that anomalies of the extracellular matrix protein fibrillin-1 with associated increased production and activation of local TGF- $\beta$ similar to what is observed in MFS may also lead to increased susceptibility to NTM infection. Abnormal fibrillin is speculated to lead to increased local production and activation of the immunosuppressive cytokine TGF- $\beta$ through alterations in its binding with the TGF- $\beta$ - latency associated peptide complex. Indeed, in a murine model of MFS, fibrillin-1 deficient mice have been found to have increased TGF- $\beta$ levels and signaling [97]. In the context of mycobacterial infection, TGF- $\beta$ is increased in PBMCs from patients with TB disease $[98,99]$ as well as in murine models of MAC infection [100] where exogenous TGF- $\beta$ administration has also been shown to lead to progression of infection [81]. Although individuals with MFS are not generally considered to have increased risk of infection (other than endocarditis due to valvular abnormalities), innate and adaptive immune defects have been described among these individuals [101]. Thus, the constellation of fibrillin and TGF- $\beta$ abnormalities, in conjunction with estrogen deficiency and adipokine abnormalities, were hypothesized by Chan and Iseman to be interrelated in promoting NTM susceptibility among thin older women with these distinctive body morphologic features.

\section{CONCLUSIONS}

The nontuberculous mycobacteria (NTM) are an increasingly important cause of lung disease, particularly among older women. Evidence of the rising prevalence of NTM lung disease, along with the ubiquity of NTM in the environment and widespread means of frequent high-level exposure through aerosolized municipal water, make the NTM a legitimate emerging environmental and public health threat.

Although multiple factors have been hypothesized and evaluated in the causative pathway of this condition, a full understanding of the majority of NTM lung disease is still lacking. And despite the identification of predisposing conditions for this disease, the majority of individuals with such predisposition still do not develop disease. Clearly there are additional unidentified host and/or pathogen factors involved in NTM lung disease pathogenesis.

In particular, many predisposing conditions for NTM lung disease that have been identified to date, such as chronic lung disease and estrogen deprivation among postmenopausal women, are likely to increase in prevalence in our enlarging aged population. Thus, the incidence of NTM disease may continue to rise in the coming years. Further research to identify predisposing factors and mechanisms of disease progression is needed in order for appropriate identification of at-risk individuals and disease prevention efforts.

\section{CONFLICT OF INTEREST}

None declared.

\section{ACKNOWLEDGEMENTS}

None declared.

\section{REFERENCES}

[1] Griffith DE. An official ATS/IDSA statement: diagnosis, treatment, and prevention of nontuberculous mycobacterial diseases. Am J Respir Crit Care Med 2007; 175(4): 367-416. 
[2] Marras TK, Daley CL. Epidemiology of human pulmonary infection with nontuberculous mycobacteria. Clin Chest Med 2002; 23(3): 553-67.

[3] Brown-Elliot BA, Wallace RJ. Infections due to nontuberculous Mycobacteria other than mycobacterium avium-intracellulzare. In: Mandell GL, Ed. Principles and practice of infectious diseases. Philadelphia: Churchill Livingstone, Elsevier 2010; pp. 3191-8.

[4] Bastian S. Assessment of clarithromycin susceptibility in strains belonging to the Mycobacterium abscessus group by erm(41) and rrl sequencing. Antimicrob Agents Chemother 2011; 55(2): 775-81.

[5] von Reyn CF. Isolation of Mycobacterium avium complex from water in the United States, Finland, Zaire, and Kenya. J Clin Microbiol 1993: 31(12): 3227-30.

[6] Falkinham JO, $3^{\text {rd }}$. Nontuberculous mycobacteria in the environment. Clin Chest Med 2002; 23(3): 529-51.

[7] Feazel LM. Opportunistic pathogens enriched in showerhead biofilms. Proc Natl Acad Sci USA 2009; 106(38): 16393-9.

[8] Falkinham JO, $3^{\text {rd }}$. Nontuberculous mycobacteria from household plumbing of patients with nontuberculous mycobacteria disease. Emerg Infect Dis 2011; 17(3): 419-24.

[9] Marras TK. Hypersensitivity pneumonitis reaction to Mycobacterium avium in household water. Chest 2005; 127(2): 664-71.

[10] Falkinham JO. Mycobacterium avium in a shower linked to pulmonary disease. J Water Health 2008; 6(2): 209-13.

[11] Timpe A, Runyon EH. The relationship of atypical acid-fast bacteria to human disease; a preliminary report. J Lab Clin Med 1954; 44(2): 202-9.

[12] Crow HE. A limited clinical, pathologic, and epidemiologic study of patients with pulmonary lesions associated with atypical acidfast bacilli in the sputum. Am Rev Tuberc 1957; 75(2): 199-222.

[13] O'Brien RJ, Geiter LJ, Snider DE Jr. The epidemiology of nontuberculous mycobacterial diseases in the United States. Results from a national survey. Am Rev Respir Dis 1987; 135(5): 1007-14.

[14] Marras TK. Isolation prevalence of pulmonary non-tuberculous mycobacteria in Ontario, 1997 2003. Thorax 2007; 62(8): 661-6.

[15] Cassidy PM. Nontuberculous mycobacterial disease prevalence and risk factors: a changing epidemiology. Clin Infect Dis 2009; 49(12): e124-9.

[16] Winthrop KL. Pulmonary nontuberculous mycobacterial disease prevalence and clinical features: an emerging public health disease. Am J Respir Crit Care Med 2010; 182(7): 977-82.

[17] Prevots DR. Nontuberculous mycobacterial lung disease prevalence at four integrated health care delivery systems. Am J Respir Crit Care Med 2010; 182(7): 970-6.

[18] Falkinham JO. Epidemiology of infection by nontuberculous mycobacteria. Clin Microbiol Rev 1996; 9(2): 177-215.

[19] Griffith DE, Girard WM, Wallace RJ. Clinical features of pulmonary disease caused by rapidly growing mycobacteria. An analysis of 154 patients. Am Rev Respir Dis 1993; 147(5): 1271-8.

[20] Witty LA, Tapson VF. Piantadosi, Isolation of mycobacteria in patients with pulmonary alveolar proteinosis. Medicine (Baltimore) 1994; 73(2): 103-9.

[21] Olivier KN. Nontuberculous mycobacteria. I: multicenter prevalence study in cystic fibrosis. Am J Respir Crit Care Med 2003; 167(6): 828-34.

[22] Wickremasinghe M. Non-tuberculous mycobacteria in patients with bronchiectasis. Thorax 2005; 60(12): 1045-51.

[23] Morita H. Isolation of nontuberculous mycobacteria from patients with pneumoconiosis. J Infect Chemother 2005; 11(2): 89-92.

[24] Fowler SJ. Nontuberculous mycobacteria in bronchiectasis: Prevalence and patient characteristics. Eur Respir J 2006; 28(6): 1204-10.

[25] Reich JM, Johnson RE. Mycobacterium avium complex pulmonary disease presenting as an isolated lingular or middle lobe pattern. The Lady Windermere syndrome. Chest 1992; 101(6): 1605-9.

[26] Iseman MD, Buschman DL, Ackerson LM. Pectus excavatum and scoliosis. Thoracic anomalies associated with pulmonary disease caused by Mycobacterium avium complex. Am Rev Respir Dis 1991; 144(4): 914-6.

[27] Kim RD. Pulmonary nontuberculous mycobacterial disease: prospective study of a distinct preexisting syndrome. Am J Respir Crit Care Med 2008; 178(10): 1066-74.

[28] Okumura M. Clinical factors on cavitary and nodular bronchiectatic types in pulmonary Mycobacterium avium complex disease. Intern Med 2008; 47(16): 1465-72.
[29] Jeong YJ. Nontuberculous mycobacterial pulmonary infect immunocompetent patients: comparison of thin-section CT and histopathologic findings. Radiology 2004; 231(3): 880-6.

[30] Holland SM. Nontuberculous mycobacteria. Am J Med Sci 2001; 321(1): 49-55.

[31] Haverkamp MH, van Dissel JT, Holland SM. Human host genetic factors in nontuberculous mycobacterial infection: lessons from single gene disorders affecting innate and adaptive immunity and lessons from molecular defects in interferon-gamma-dependent signaling. Microbes Infect 2006; 8(4): 1157-66.

[32] Houben EN, Nguyen L, Pieters J. Interaction of pathogenic mycobacteria with the host immune system. Curr Opin Microbiol 2006; 9(1): 76-85.

[33] Guide SV, Holland SM. Host susceptibility factors in mycobacterial infection. Genetics and body morphotype. Infect Dis Clin North Am 2002; 16(1): 163-86.

[34] Picard C. Inherited interleukin-12 deficiency: IL12B genotype and clinical phenotype of 13 patients from six kindreds. Am J Hum Genet 2002; 70(2): 336-48.

[35] Dorman SE, Holland SM. Mutation in the signal-transducing chain of the interferon-gamma receptor and susceptibility to mycobacterial infection. J Clin Invest 1998; 101(11): 2364-9.

[36] Fieschi C. Low penetrance, broad resistance, and favorable outcome of interleukin 12 receptor beta1 deficiency: medical and immunological implications. J Exp Med 2003; 197(4): 527-35.

[37] Rosenzweig SD. A novel mutation in IFN-gamma receptor 2 with dominant negative activity: biological consequences of homozygous and heterozygous states. J Immunol 2004; 173(6): 4000-8.

[38] van de Vosse E, Hoeve MA, Ottenhoff TH. Human genetics of intracellular infectious diseases: molecular and cellular immunity against mycobacteria and salmonellae. Lancet Infect Dis 2004; 4(12): 739-49.

[39] Dupuis S. Impairment of mycobacterial but not viral immunity by a germline human STAT1 mutation. Science 2001; 293(5528): 3003.

[40] Patel SY. Anti-IFN-gamma autoantibodies in disseminated nontuberculous mycobacterial infections. J Immunol 2005; 175(7): 4769-76.

[41] Kampitak T. Anti-interferon-gamma autoantibody and opportunistic infections: case series and review of the literature. Infection 2011; 39(1): 65-71.

[42] Salama C, Policar M, Venkataraman M. Isolated pulmonary Mycobacterium avium complex infection in patients with human immunodeficiency virus infection: case reports and literature review. Clin Infect Dis 2003; 37(3): e35-40.

[43] Doucette K, Fishman JA. Nontuberculous mycobacterial infection in hematopoietic stem cell and solid organ transplant recipients. Clin Infect Dis 2004; 38(10): 1428-39.

[44] Wallis RS. Infectious complications of tumor necrosis factor blockade. Curr Opin Infect Dis 2009; 22(4): 403-9.

[45] Winthrop KL. Nontuberculous mycobacteria infections and antitumor necrosis factor-alpha therapy. Emerg Infect Dis 2009; 15(10): 1556-61.

[46] Gomes MS, Flórido M, Cordeiro JV, et al. Limited role of the Tolllike receptor-2 in resistance to Mycobacterium avium. Immunology 2004; 111(2): 179-85.

[47] Ryu YJ, Kim EJ, Lee SH, et al. Impaired expression of Toll-like receptor 2 in nontuberculous mycobacterial lung disease. Eur Respir J 2007; 30(4): 736-42.

[48] Koh WJ, Kwon OJ, Kim EJ, et al. NRAMP1 gene polymorphism and susceptibility to nontuberculous mycobacterial lung diseases. Chest 2005; 128(1): 94-101.

[49] Kwon YS, Kim EJ, Lee SH, et al. Decreased cytokine production in patients with nontuberculous mycobacterial lung disease. Lung 2007; 185(6): 337-41.

[50] Greinert U, Schlaak M, Rüsch-Gerdes S, Flad HD, Ernst M. Low in vitro production of interferon-gamma and tumor necrosis factoralpha in HIV-seronegative patients with pulmonary disease caused by nontuberculous mycobacteria. J Clin Immunol 2000; 20(6): 44552.

[51] Vankayalapati R, Wizel B, Samten B, et al. Cytokine profiles in immunocompetent persons infected with Mycobacterium avium complex. J Infect Dis 2001; 183(3): 478-84. 
[52] Safdar A, White DA, Stover D. Profound interferon gamma deficiency in patients with chronic pulmonary nontuberculous mycobacteriosis. Am J Med 2002; 113(9): 756-9.

[53] Safdar A, Armstrong D, Murray HW. A novel defect in interferongamma secretion in patients with refractory nontuberculous pulmonary mycobacteriosis. Ann Intern Med 2003; 138(6): 521.

[54] Huang JH, Oefner PJ, Adi V, et al. Analyses of the NRAMP1 and IFN-gammaR1 genes in women with Mycobacterium aviumintracellulare pulmonary disease. Am J Respir Crit Care Med 1998; 157(2): 377-81.

[55] Tanaka E, Amitani R, Niimi A, Suzuki K, Murayama T, Kuze F. Yield of computed tomography and bronchoscopy for the diagnosis of Mycobacterium avium complex pulmonary disease. Am J Respir Crit Care Med 1997; 155(6): 2041-6.

[56] Nnoaham KE, Clarke A. Low serum vitamin D levels and tuberculosis: a systematic review and meta-analysis. Int J Epidemiol 2008; 37(1): 113-9.

[57] Gao L, Tao Y, Zhang L, Jin Q. Vitamin D receptor genetic polymorphisms and tuberculosis: updated systematic review and meta-analysis. Int J Tuberc Lung Dis 2010; 14(1): 15-23.

[58] Gelder CM, Hart KW, Williams OM, et al. Vitamin D receptor gene polymorphisms and susceptibility to Mycobacterium malmoense pulmonary disease. J Infect Dis 2000; 181(6): 2099102.

[59] Tanaka G, Shojima J, Matsushita I, et al. Pulmonary Mycobacterium avium complex infection: association with NRAMP1 polymorphisms. Eur Respir J 2007; 30(1): 90-6.

[60] Middleton AM, Chadwick MV, Nicholson AG, et al. The role of Mycobacterium avium complex fibronectin attachment protein in adherence to the human respiratory mucosa. Mol Microbiol 2000; 38(2): 381-91.

[61] Middleton AM, Chadwick MV, Nicholson AG, et al. Inhibition of adherence of Mycobacterium avium complex and Mycobacterium tuberculosis to fibronectin on the respiratory mucosa. Respir Med 2004; 98(12): 1203-6

[62] Martín-de-Hijas NZ, García-Almeida D, Ayala G. Biofilm development by clinical strains of non-pigmented rapidly growing mycobacteria. Clin Microbiol Infect 2009; 15(10): 931-6.

[63] Morrissey BM. Pathogenesis of bronchiectasis. Clin Chest Med 2007; 28(2): 289-96.

[64] Casals T, De-Gracia J, Gallego M, et al. Bronchiectasis in adult patients: an expression of heterozygosity for CFTR gene mutations? Clin Genet 2004; 65(6): 490-5.

[65] King PT, Freezer NJ, Holmes PW, Holdsworth SR, Forshaw K, Sart DD. Role of CFTR mutations in adult bronchiectasis. Thorax 2004. 59(4): 357-8.

[66] Divac A, Nikolic A, Mitic-Milikic M, et al. CFTR mutations and polymorphisms in adults with disseminated bronchiectasis: a controversial issue. Thorax 2005; 60(1): 85.

[67] Bienvenu T, Sermet-Gaudelus I, Burge PR, et al. Cystic fibrosis transmembrane conductance regulator channel dysfunction in noncystic fibrosis bronchiectasis. Am J Respir Crit Care Med 2010; 181(10): 1078-84.

[68] Ziedalski TM, Kao PN, Henig NR, Jacobs SS, Ruoss SJ. Prospective analysis of cystic fibrosis transmembrane regulator mutations in adults with bronchiectasis or pulmonary nontuberculous mycobacterial infection. Chest 2006; 130(4): 9951002.

[69] Colombo RE, Hill SC, Claypool RJ, et al. Familial clustering of pulmonary nontuberculous mycobacterial disease. Chest 2010; 137(3): 629-34.

[70] PG Noone, Leigh MW, Sannuti A, et al. Primary ciliary dyskinesia: diagnostic and phenotypic features. Am J Respir Crit Care Med 2004; 169(4): 459-67.

[71] Olivier KN, O'Connell ML, Holland SM, Knowles MR. Mucosal defense abnormalities in idiopathic bronchiectasis associated with nontuberculous Mycobacteria. American Thoracic Society International Conference; 2010 May 14-19; New Orleans, LA, USA.

[72] Lieberman J. Augmentation therapy reduces frequency of lung infections in antitrypsin deficiency: a new hypothesis with supporting data. Chest 2000; 118(5): 1480-5.

[73] Chan ED, Kaminska AM, Gill W, et al. Alpha-1-antitrypsin (AAT) anomalies are associated with lung disease due to rapidly growing mycobacteria and AAT inhibits Mycobacterium abscessus infection of macrophages. Scand J Infect Dis 2007; 39(8): 690-6.
[74] Prince DS, Peterson DD, Steiner RM, et al. Infection with Mycobacterium avium complex in patients without predisposing conditions. N Engl J Med 1989; 321(13): 863-8.

[75] Rubin BK. Did Lady Windermere have cystic fibrosis? Chest 2006; 130(4): 937-8

[76] Chan ED, Iseman MD. Older women appear to be more susceptible to nontuberculous mycobacterial lung disease. Gend Med 2010; 7(1): 5-18.

[77] Pond CM. Paracrine interactions of mammalian adipose tissue. J Exp Zool A Comp Exp Biol 2003; 295(1): 99-110.

[78] Lord GM, Matarese G, Howard JK, et al. Leptin modulates the Tcell immune response and reverses starvation-induced immunosuppression. Nature 1998; 394(6696): 897-901.

[79] Wieland CW, Florquin S, Chan ED, et al. Pulmonary Mycobacterium tuberculosis infection in leptin-deficient ob/ob mice. Int Immunol 2005; 17(11): 1399-408.

[80] Ordway D, Henao-Tamayo M, Smith E, et al. Animal model of Mycobacterium abscessus lung infection. J Leukoc Biol 2008; 83(6): 1502-11.

[81] Denis M, Ghadirian E. Interleukin-1 is involved in mouse resistance to Mycobacterium avium. Infect Immun 1994; 62(2): 457-61.

[82] Beamer GL, Flaherty DK, Assogba BD, et al. Interleukin-10 promotes Mycobacterium tuberculosis disease progression in CBA/J mice. J Immunol 2008; 181(8): 5545-50.

[83] Tasaka S, Hasegawa N, Nishimura $\mathrm{T}$, et al. Elevated serum adiponectin level in patients with Mycobacterium aviumintracellulare complex pulmonary disease. Respiration 2010; 79(5): 383-7.

[84] Grossman CJ. Interactions between the gonadal steroids and the immune system. Science 1985; 227(4684): 257-61.

[85] Whitacre CC. Sex differences in autoimmune disease. Nat Immunol 2001; 2(9): 777-80.

[86] Bouman A, Heineman MJ, Faas MM. Sex hormones and the immune response in humans. Hum Reprod Update 2005; 11(4): 411-23.

[87] Marriott I, Huet-Hudson YM. Sexual dimorphism in innate immune responses to infectious organisms. Immunol Res 2006; 34(3): 177-92.

[88] Neyrolles O, Quintana-Murci L. Sexual inequality in tuberculosis PLoS Med 2009; 6(12): e1000199.

[89] Chalermskulrat W, Gilbey JG, Donohue JF. Nontuberculous mycobacteria in women, young and old. Clin Chest Med 2002; 23(3): 675-86

[90] Gourdy P, Luiza M. Araujo, et al. Relevance of sexual dimorphism to regulatory T cells: estradiol promotes IFN-gamma production by invariant natural killer T cells. Blood 2005; 105(6): 2415-20.

[91] Yamamoto Y, Tomioka H, Sato K, et al. Sex differences in the susceptibility of mice to infection induced by Mycobacterium intracellulare. Am Rev Respir Dis 1990; 142(2): 430-3.

[92] Yamamoto T, Endo S, Yokota T. Characteristics of adherence of enteroaggregative Escherichia coli to human and animal mucosa. Infect Immun 1991; 59(10): 3722-39.

[93] Gameiro C, Romao F. Changes in the immune system during menopause and aging. Front Biosci (Elite Ed) 2010; 2: 1299-303.

[94] Tsuyuguchi K, Suzuki K, Matsumoto H, et al. Effect of oestrogen on Mycobacterium avium complex pulmonary infection in mice. Clin Exp Immunol 2001; 123(3): 428-34.

[95] Lambert KC, Curran EM, Judy BM, Lubahn DB, Estes DM. Estrogen receptor-alpha deficiency promotes increased TNF-alpha secretion and bacterial killing by murine macrophages in response to microbial stimuli in vitro. J Leukoc Biol 2004. 75(6): 1166-72.

[96] Curran EM, Judy BM, Newton LG. Dietary soy phytoestrogens and ERalpha signalling modulate interferon gamma production in response to bacterial infection. Clin Exp Immunol 2004; 135(2): 219-25.

[97] Neptune ER, Frischmeyer PA, Arking DE, et al. Dysregulation of TGF-beta activation contributes to pathogenesis in Marfan syndrome. Nat Genet 2003; 33(3): 407-11.

[98] Hirsch CS, Ellner JJ, Blinkhorn R, Toossi Z. In vitro restoration of $\mathrm{T}$ cell responses in tuberculosis and augmentation of monocyte effector function against Mycobacterium tuberculosis by natural inhibitors of transforming growth factor beta. Proc Natl Acad Sci USA 1997; 94(8): 3926-31.

[99] Roberts T, Beyers N, Aguirre A, et al. Immunosuppression during active tuberculosis is characterized by decreased interferon- gamma 
production and CD25 expression with elevated forkhead box P3, transforming growth factor- beta, and interleukin-4 mRNA levels. J Infect Dis 2007; 195(6): 870-8.

[100] Champsi J, Young LS, Bermudez LE. Production of TNF-alpha, IL-6 and TGF-beta, and expression of receptors for TNF-alpha and
IL-6, during murine Mycobacterium avium infection. Immunology 1995; 84(4): 549-54.

[101] Smith TF, Engel E. Marfan's syndrome with 47,XXX genotype and possible immunologic abnormality. South Med J 1981; 74(5): 6302.

Received: June 24, 2011

(C) Epson and Winthrop; Licensee Bentham Open.

This is an open access article licensed under the terms of the Creative Commons Attribution Non-Commercial License (http://creativecommons.org/licenses/by-nc/3.0/) which permits unrestricted, non-commercial use, distribution and reproduction in any medium, provided the work is properly cited. 